\title{
MicroRNA-132 suppresses cell proliferation in human breast cancer by directly targeting FOXA1
}

\author{
Dan WANG ${ }^{1}$, Jin REN ${ }^{2}$, Hui REN ${ }^{3}$, Jin-ling $\mathrm{FU}^{4, *}$, Dan $\mathrm{YU}^{5, *}$ \\ ${ }^{1}$ Department of Breast Surgery; ${ }^{2}$ Department of Respiratory Medicine; ${ }^{3}$ Department of Colorectal Surgery; ${ }^{4}$ Department of \\ Ophthalmology; ${ }^{5}$ Department of Otolaryngology Head and Neck Surgery, the Second Hospital of Jilin University, Changchun 130041, \\ China
}

\begin{abstract}
Dysregulation of microRNAs (miRNAs) has been implicated in cancer. Recently, miR-132 has been reported to be downregulated in the tissues of patients with breast cancer. In this study, we investigated the functional role of miR-132 and its direct target FOXA1 in breast cancer cells. In 30 human breast cancer tissues, FOXA1 was significantly overexpressed and negatively correlated with miR-132 expression. A bioinformatics analysis suggested that FOXA1 was a potential target of miR-132. Furthermore, dual luciferase reporter assays revealed that miR-132 dose-dependently inhibited the luciferase activity of the wt 3'UTR of FOXA1 rather than the mut 3'UTR of FOXA1 in human MDA-MB-468 and SK-BR3 breast cancer cells. Moreover, ectopic miR-132 expression significantly inhibited FOXA1 protein expression, whereas miR-132 knockdown promoted FOXA1 expression in the breast cancer cells. Ectopic miR-132 expression also suppressed proliferation of the breast cancer cells, whereas miR-132 knockdown promoted proliferation of the breast cancer cells, which was reversed by knockdown of FOXA1 expression. We conclude that MiR-132 suppresses proliferation of breast cancer cells at least partially though inhibition of FOXA1. These results suggest that miR-132 and FOXA1 may be potential biomarkers or therapeutic targets in breast cancer.
\end{abstract}

Keywords: breast cancer; MiR-132; FOXA1; cell proliferation

Acta Pharmacologica Sinica (2018) 39: 124-131; doi: 10.1038/aps.2017.89; published online 17 Aug 2017

\section{Introduction}

Breast cancer is the most common malignancy and the leading cause of cancer related death in females worldwide, accounting for $23 \%$ of the total new cancer cases and $14 \%$ of total cancer deaths in $2008^{[1,2]}$. Although great advances in early diagnosis and targeted therapies have been achieved in recent years, the precise causes of breast cancer remain to be fully characterized $^{[3]}$. Therefore, studies to determine the molecular mechanism underlying tumorigenesis and progression of breast cancer are greatly needed to achieve better diagnosis, prognosis, and treatment in the future.

Breast cancer is one of the representative hormone-related malignancies in humans, and dysregulation of estrogen receptor (ER) activity plays a critical role in approximately $75 \%$ of all breast cancer ${ }^{[4]}$. As a result, inhibitors against the ER are commonly used in breast cancer treatment. Transcriptional

\footnotetext{
* To whom correspondence should be addressed.

E-mail wangdan79@126.com (Dan YU); fujinling1981@163.com (Jin-ling FU)

Received 2017-02-09 Accepted 2017-05-19
}

regulation by the ER is associated with altered expression signatures in breast cancer, owing to increased ER activity. Recruitment of the ER to chromatin requires FOXA1 ${ }^{[4]}$, which is termed a "pioneer factor" that regulates chromatin accessibility and thereby enables ER-dependent gene activation and proliferation of breast cancer cells ${ }^{[5]}$. FOXA1 belongs to the FOXA gene family, which has previously been reported to be a cancer-defining transcription factor in luminal breast cancer $^{[6,7]}$. Increasing evidence indicates that FOXA1 plays an oncogenic role, thereby regulating proliferation, apoptosis and the cell cycle, and predicts poor overall survival in anaplastic thyroid cancer ${ }^{[8]}$, prostate cancer ${ }^{[9]}$ and breast cancer ${ }^{[10]}$. Cumulatively, these reports suggest that FOXA1 is a crucial player in ER functionality in hormone-related cancer, especially breast cancer. However, a recent study has demonstrated that FOXA1 is highly expressed in $\mathrm{ER}^{+}$breast cancer cells (MCF7, T47D, BT474 and ZR75), the ER /HER2 $^{+}$SK-BR3 and triple negative-like MDA-MB-468 cell lines. In all breast cancer subtypes, FOXA1 or FOXA2 controls the endothelial lipase LIPG, which is ubiquitously and highly expressed. Thus, FOXA regulation of LIPG controls the uptake of extracellular lipids, 
thereby supporting breast cancer growt $\mathrm{h}^{[11]}$. The regulation of FOXA1 expression in breast cancer remains to be fully characterized, especially regulation by microRNAs (miRNAs).

MiRNAs are small RNAs with no coding potential that negatively regulate protein-coding gene expression by promotion of mRNA degradation or inhibition of translation ${ }^{[12,13]}$. The involvement of miRNAs in cancer biology processes has been demonstrated by numerous studies, and dysregulation of various miRNAs, either downregulation or upregulation, have been reported in breast cancer ${ }^{[14]}$. Aberrant expression of cancer-associated genes induced by miRNAs, through upregulation of oncogenes or downregulation of tumor suppressors, plays critical roles in cancer initiation and progression in almost all types of tumors, including breast cancer ${ }^{[15-19]}$.

MiR-132, which was first reported in the inflammatory context and is upregulated by pro-inflammatory signals ${ }^{[20]}$, has been reported to play important roles in tumorigenesis and progression in regulating cell proliferation, cell cycle, apoptosis, migration, invasion and angiogenesis ${ }^{[21-25]}$. MiR-132 is downregulated in several cancers, including breast cancer ${ }^{[21]}$, prostate cancer ${ }^{[26]}$, colorectal cancer ${ }^{[22]}$, osteosarcoma ${ }^{[27]}$, nonsmall cell lung cancer ${ }^{[24,28]}$, and hepatocellular carcinoma ${ }^{[29-31]}$. Beyond several reported target genes of miR-132, downregulation of miR-132 has also been reported to be associated with aberrant promoter methylation ${ }^{[32,33]}$. Recently, a report has demonstrated that miR-132 expression is downregulated in tissues of patients with breast cancer ${ }^{[34]}$, and this decreased expression is most prominent in high-grade tumors ${ }^{[34]}$. However, the biological roles and potential molecular mechanism of miR-132 in breast cancer remain largely unclear. In this study, FOXA1 was found to be a direct target of miR-132 by prediction. Therefore, the aim of the present study was to determine the role and potential mechanisms of miR-132, including its direct target FOXA1, in breast cancer.

\section{Materials and methods}

\section{Patients and tissue samples}

Breast cancer tissue samples and corresponding adjacent normal tissue samples were obtained from 30 patients with primary breast cancer who underwent surgery at the Second Hospital of Jilin University between January 2012 and October 2015. Detailed clinical information of enrolled patients is listed in Supplementary Table S1. All tissue samples were immediately frozen in liquid nitrogen and stored at $-80{ }^{\circ} \mathrm{C}$ until RNA extraction. None of the patients recruited in this study had undergone preoperative chemotherapy, radio-therapy or other therapy. Informed consent was obtained from all patients before surgery. This study was approved by the ethics committee of the Second Hospital of Jilin University.

\section{RNA isolation and quantitative qRT-PCR}

Total RNA was extracted from cells by using TRIzol reagent (Invitrogen, Carlsbad, CA, USA) according to the manufacturer's protocol. Total RNA was extracted from frozen tissues (up to $30 \mathrm{mg}$ each) using an miRNeasy Minikit (Qiagen, Valencia, CA, USA). For FOXA1 expression, first strand
cDNA synthesis was prepared using PrimeScript RT Master Mix (TaKaRa Biotechnology, Dalian, China), according to the manufacturer's instructions. qRT-PCR was performed using a QuantiNova SYBR Green PCR kit (Qiagen, Valencia, CA, USA). Relative FOXA1 mRNA levels were analyzed by normalizing the threshold cycle $(\mathrm{Ct})$ value to that of the internal loading control GAPDH.

To quantify miR-132, miR-132 expression was measured using a TaqMan MicroRNA Reverse Transcription Kit (Thermo Fisher, Carlsbad, CA, USA) and TaqMan gene expression master mix (Thermo Fisher, Carlsbad, CA, USA) according to the manufacturer's protocol. U6 snRNA was used as an internal loading control. All PCR reactions were performed in triplicate for each sample. PCR primers used in this study are listed in Supplementary Table S2.

\section{Cell culture}

MDA-MB-468 and SK-BR3 human breast cancer cell lines were acquired from the American Type Culture Collection (ATCC, Manassas, VA, USA). Breast cancer cells were maintained in RPMI-1640 (Invitrogen, Carlsbad, CA, USA) medium supplemented with $10 \%$ fetal bovine serum (FBS). Cells were cultured in a humidified incubator at $37^{\circ} \mathrm{C}$ with $5 \% \mathrm{CO}_{2}$.

\section{Transfection of microRNA mimics, inhibitor and siRNA}

The miR-132 mimics, inhibitor (anti-miR-132) oligonucleotides and siRNAs (si-FOXA1) were purchased from GenePharma (Shanghai, China). The miR-132 mimics, anti-miR-132 and negative controls were transfected into cells at $50 \mathrm{nmol} / \mathrm{L}$ and $100 \mathrm{nmol} / \mathrm{L}$ concentrations with Lipofectamine 2000 (Invitrogen, Carlsbad, CA, USA) according to the manufacturer's instructions. Sequences for siRNAs are listed in Supplementary Table S2.

\section{Cell proliferation assay}

For cell proliferation assays, transfected cells were plated into 96-well plates and cultured for 24-72 h. At the indicated times, $10 \mu \mathrm{L}$ MTT (Thermo Fisher) was added to each well, and cells were cultured for an additional $2 \mathrm{~h}$. The absorbance was then detected at a wavelength of $450 \mathrm{~nm}$.

For colony formation assays, twenty-four hours after transfection with miR-132 mimics, anti-miR-132, si-FOXA1 or negative control oligonucleotides, breast cancer cells were seeded onto six-well plates at a density of 500 cells per well and cultured for 10 days. Then, colonies were fixed with methanol and stained with $1 \%$ crystal violet (Sigma), and the number of colonies was then counted.

\section{Western blotting}

Seventy-two hours after transfection, cells were harvested with RIPA lysis buffer. Protein lysates were separated using 12\% SDS-PAGE and transferred to PVDF membranes (Millipore, Billerica, MA, USA). The membranes were incubated with anti-FOXA1 (Cell Signaling Technology, MA, USA), antiLIPG (Abcam, Cambridge, USA) or anti- $\beta$-actin (Cell Signaling Technology, MA, USA), then with HRP-labeled goat anti 
rabbit IgG or anti-mouse IgG. The density of the bands was quantified using ImageQuant 5.2 software (GE Healthcare, Little Chalfont, UK).

\section{Dual luciferase reporter assay}

A sequence of FOXA1 3'UTR containing the predicted miR132 binding site and a sequence of FOXA1 3'UTR with five mutations in the putative miR-132 binding site was cloned into the pGL3 luciferase reporter vector (Promega, Madison, MI, USA). Primers were listed in Supplementary Table S2. Then, cells seeded in 6-well plates were co-transfected with miR-132 mimics, anti-miR-132 or negative control and reporter constructs $(0.5 \mu \mathrm{g})$ by using Lipofectamine 2000 . Cell extracts were prepared $48 \mathrm{~h}$ after transfection, and luciferase activity was measured using the Dual-Luciferase Reporter Assay System (Promega).

\section{Statistical analysis}

The statistical significance between two groups was measured using Student's $t$-test. One-way analysis of variance (ANOVA) was used to measure the significance of comparisons for more than two groups. All statistical analysis and plots were performed and generated using GraphPad Prism software (version 5.01; GraphPad Software, Inc, CA, USA).
The data are presented as the mean values with standard error of the mean (SEM), and a $P$-value less than 0.05 was considered statistically significant. All experiments were performed independently at least three times.

\section{Results}

FOXA1 negatively correlates with miR-132 in breast cancer tissues

The expression levels of FOXA1 and miR-132 were detected in 30 paired frozen human breast cancer tissues and peritumor tissues by qRT-PCR. FOXA1 mRNA expression in breast cancer tissues was significantly higher than that in peritumor tissues $(P<0.01)$ (Figure $1 \mathrm{~A})$, a result consistent with those from previous reports. To investigate and validate the potential relationship between FOXA1 and miR-132, correlation analysis was also conducted, and the results demonstrated that expression of miR-132 was negatively correlated with FOXA1 expression in 30 breast cancer tissues $(r=-0.946, P<0.01)$ (Figure 1B). Furthermore, immunohistochemistry assays of FOXA1 revealed positive expression in human breast tissues (Figure 1C). Among the 30 breast cancer tissues, $73.3 \%$ of the patients $(22 / 30)$ exhibited positive expression of FOXA1, whereas $53.3 \%$ of the patients $(16 / 30)$ exhibited a strong positive FOXA1 signal (Figure 1C). These findings suggested that
A

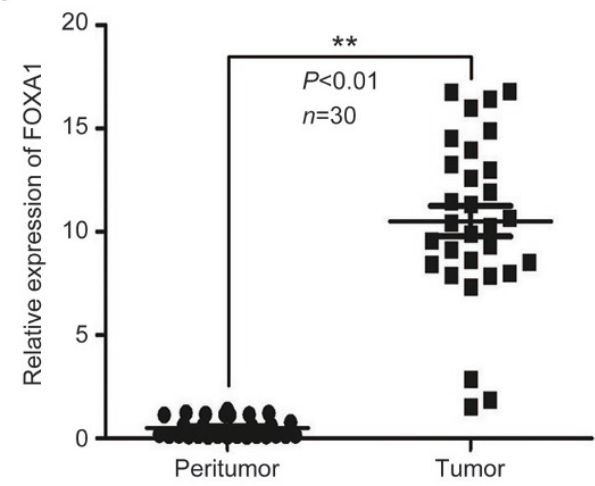

B

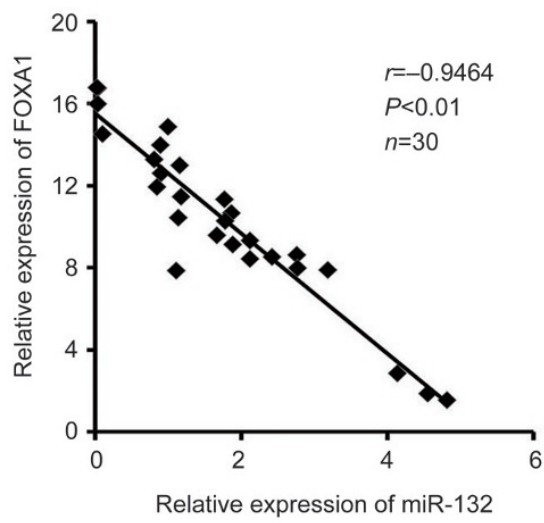

C

Negative control

$\mathrm{T} 1$

$\mathrm{T} 2$

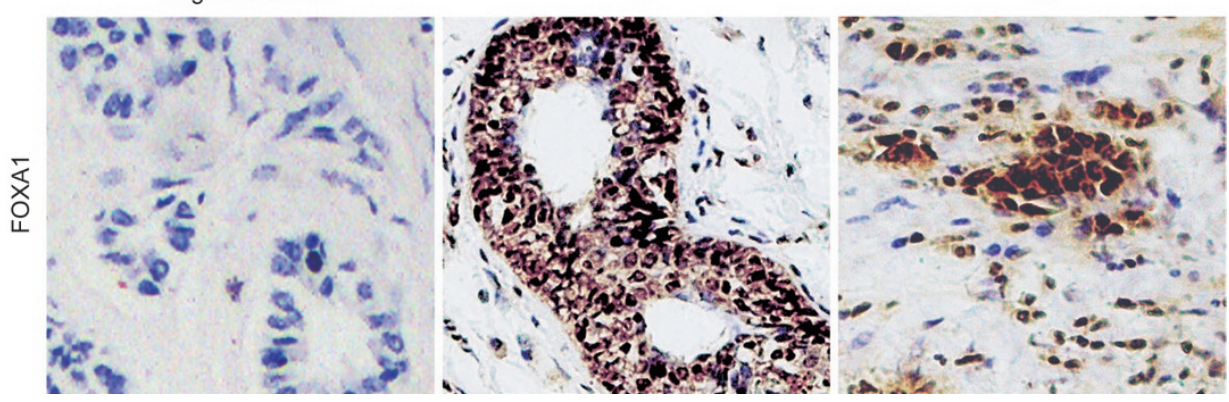

Figure 1. FOXA1 expression is negatively correlated with miR-132 in clinical breast cancer tissues. (A) FOXA1 was significantly overexpressed in 30 breast cancer tissues, as assessed by qRT-PCR. (B) MiR-132 was negatively correlated with FOXA1 expression in breast cancer tissues. (C) Immunochemistry detection of FOXA1 expression in 30 breast cancer tissues. Here, $73.3 \%$ of the patients (22/30) exhibited positive FOXA1 expression, whereas $53.3 \%$ of the patients $(16 / 30)$ exhibited strong positive FOXA1 signals. ${ }^{* *} P<0.01$. 
FOXA1 might be a direct target of miR-132, and upregulation of FOXA1 was promoted by decreased miR-132 expression.

\section{FOXA1 is a direct gene of miR-132}

Computational prediction of miRNA targets was conducted using the TargetScan database (http://www.targetscan. org/), and this in silico analysis also suggested that FOXA1 is a potential target of miR-132. In addition, a complementary seed region between FOXA1 and miR-132 was also generated. The conserved 7-mer site in the 3'UTR of FOXA1 and miR-132 is presented in Figure 2A. Therefore, dual luciferase reporter pGL3 vectors were constructed including both putative miR-132 binding sites (wildtype, wt) and mutated binding sites (mutational type, mut) in the 3'UTR of FOXA1 (Figure 2B). Dual luciferase reporter assays revealed that miR-132 significantly inhibited the luciferase activity of the wt $3^{\prime}$ UTR of FOXA1 rather than the mut 3'UTR of FOXA1 in a dosedependent manner, in both MDA-MB-468 and SK-BR3 cell lines (Figure 2C and D). Furthermore, knockdown of miR-132 expression significantly increased the luciferase activity of the wt 3'UTR of FOXA1 but had no inhibitory effect on the mut 3'UTR of FOXA1 in a dose-dependent manner in both breast cancer cell lines (Figure 2E and F). These results indicated that FOXA1 is a direct target of miR-132 in breast cancer cells.

\section{MiR-132 suppresses the expression of FOXA1}

To further validate that FOXA1 expression is regulated by miR-132, FOXA1 protein expression was detected by Western blotting in the context of interference by miR-132 in breast cancer cells. As shown in Figure 3, overexpression of miR-132 and knockdown of miR-132 in MDA-MB-468 and SK-BR3 cells was generated by transient transfection of miR-132 mimics or anti-miR-132 at concentrations of $50 \mathrm{nmol} / \mathrm{L}$ and $100 \mathrm{nmol} / \mathrm{L}$, respectively. Consequently, ectopic miR-132 expression significantly inhibited the protein expression of FOXA1 and its downstream effector LIPG in both MDA-MB-468 and SK-BR3
A

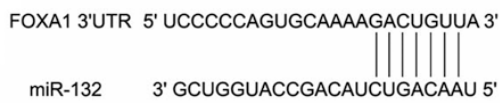

C

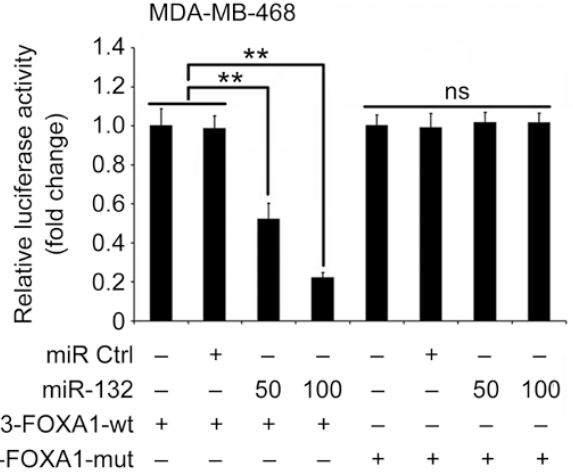

$E$

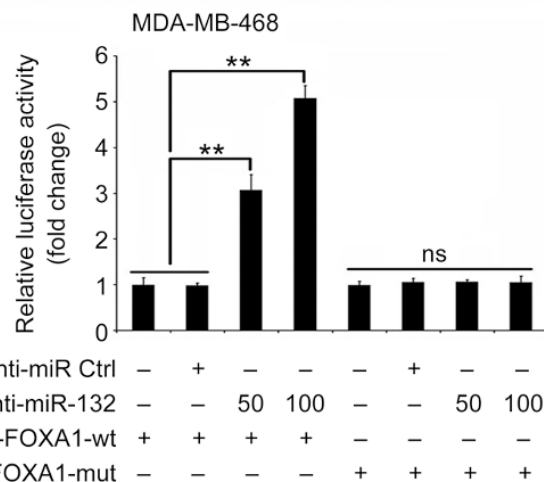

B

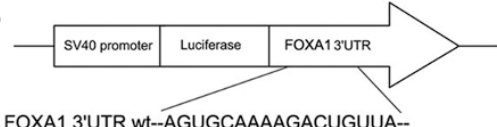

FOXA1 3'UTR wt--AGUGCA FOXA1 3'UTR mUt--AGUGCAAAACUGAGCGA--

$\mathrm{D}$

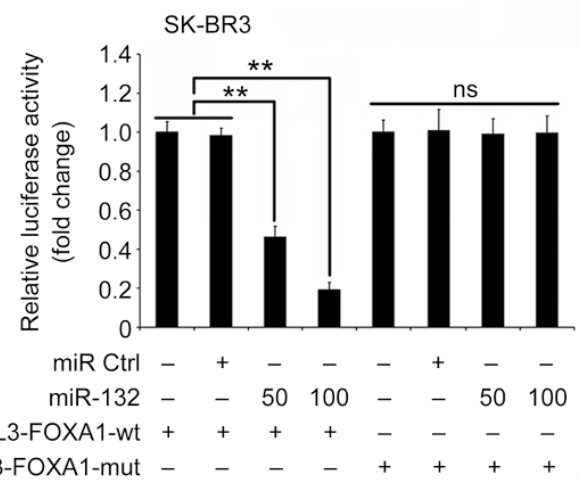

$\mathrm{F}$

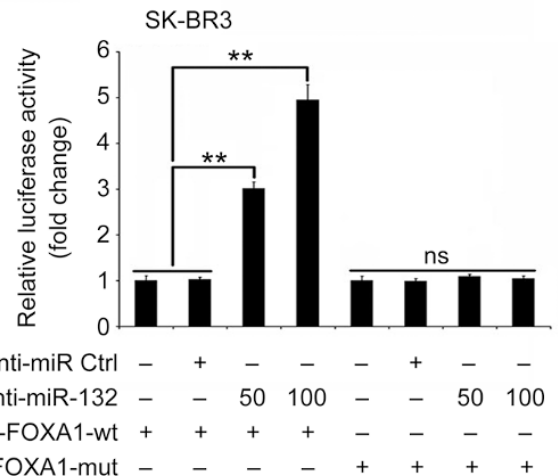

Figure 2. MiR-132 directly inhibits FOXA1 expression through targeting its 3'UTR. (A) Target prediction for miR-132 using the TargetScan database (http://www.targetscan.org). (B) Sequences for plasmid construction of the wild-type (wt) and mutated-type (mut) 3'UTR of FOXA1 mRNA. (C) and (D) MiR-132 significantly inhibited the luciferase activity of the wild-type (wt) 3'UTR of FOXA1 but had no inhibitory effect on the mutant form (mut) in a dose-dependent manner in MDA-MB-468 and SK-BR3 cell lines. ( $E$ and F) Knockdown of miR-132 by anti-miR-132 significantly promoted the luciferase activity of wild-type (wt) 3'UTR of FOXA1 but had no promoting effect on the mutant form (mut) in a dose-dependent manner in breast cancer cell lines. ${ }^{* *} P<0.01$. 
A

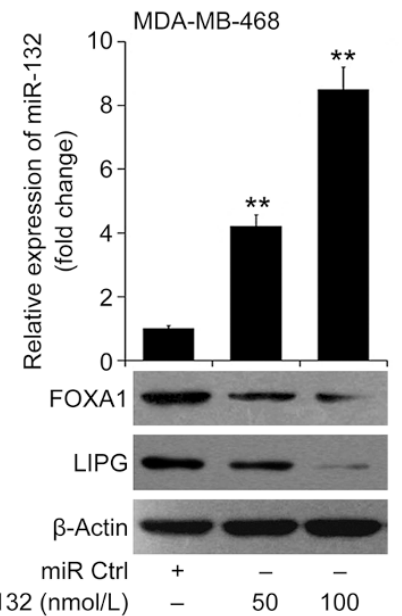

C

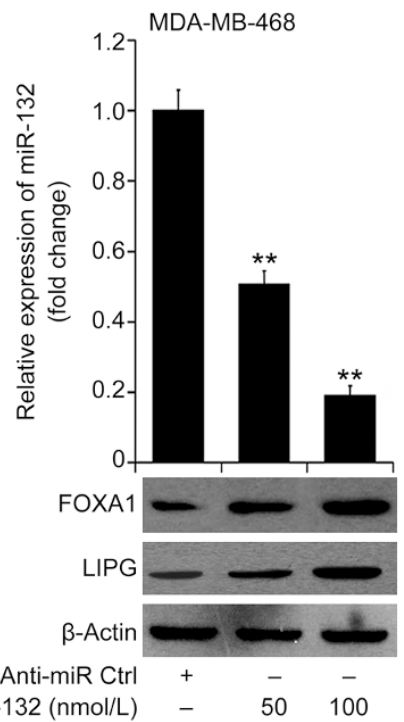

B

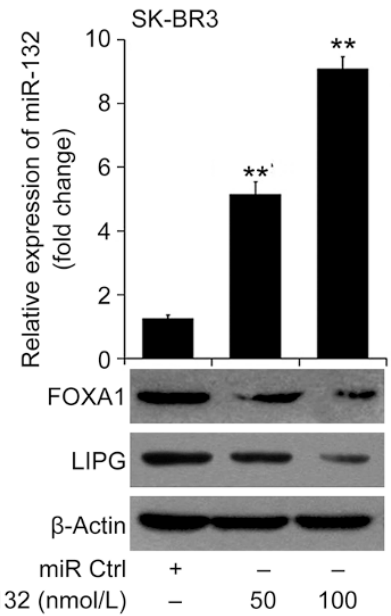

D

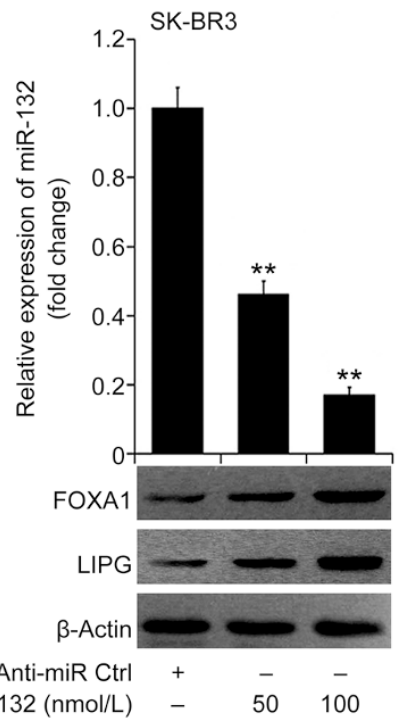

Figure 3. MiR-132 downregulates FOXA1 in breast cancer cells. (A and B) Transfection of miR-132 mimics significantly increased miR-132 expression and inhibited the protein expression of FOXA1 and its downstream effector LIPG in MDA-MB-468 and SK-BR3 cell lines in a dose-dependent manner. (C and D) Knockdown of miR-132 by anti-miR-132 significantly decreased miR-132 expression and promoted the protein expression of FOXA1 and its downstream effector LIPG in breast cancer cell lines in a dose-dependent manner. ${ }^{* *} P<0.01$.

in a dose-dependent manner (Figure $3 \mathrm{~A}$ and $\mathrm{B}$ ). In contrast, expression of FOXA1 and its downstream effector LIPG significantly increased along with knockdown of miR-132 in a dose-dependent manner in both breast cancer cells (Figure 3C and D). Together, these results demonstrated that FOXA1 is a direct target of miR-132 and that FOXA1 expression is inhibited by miR-132 in breast cancer cells.

\section{MiR-132 suppresses breast cancer cell proliferation through FOXA1}

To elucidate the functional role of miR-132 in breast cancer, cell proliferation was detected through MTT and colony formation assays in breast cancer cells that were transiently transfected with miR-132 mimics or anti-miR-132. In MTT assays, as compared with the control group, overexpression of miR132 significantly inhibited cell proliferation, whereas decreased miR-132 expression significantly promoted the growth of breast cancer cells (Figure 4A and 4B). Silencing of FOXA1 significantly suppressed breast cancer cell proliferation, as compared with the proliferation in the si-Ctrl treated group (Figure 4A and 4B). Furthermore, compared with the anti-miR-132 group, which exhibited no significant differences as compared with the control group, simultaneous knockdown of miR-132 and FOXA1 significantly decreased cell proliferation (Figure 4A and $4 \mathrm{~B})$. In colony formation assays, compared with the control group, ectopic miR-132 expression of miR-132 significantly inhibited the colony-forming activity of MDA-MB-468, whereas knockdown of miR-132 significantly promoted the colony formation efficiency of MDA-MB-468 (Figure 4C). In addition, compared with the anti-miR-132 group, inhibition of FOXA1 expression significantly decreased the colony formation efficiency of breast cancer cells (Figure 4C). These results demonstrated that knockdown of FOXA1 reversed the effect of miR-132 on the proliferation of breast cancer 

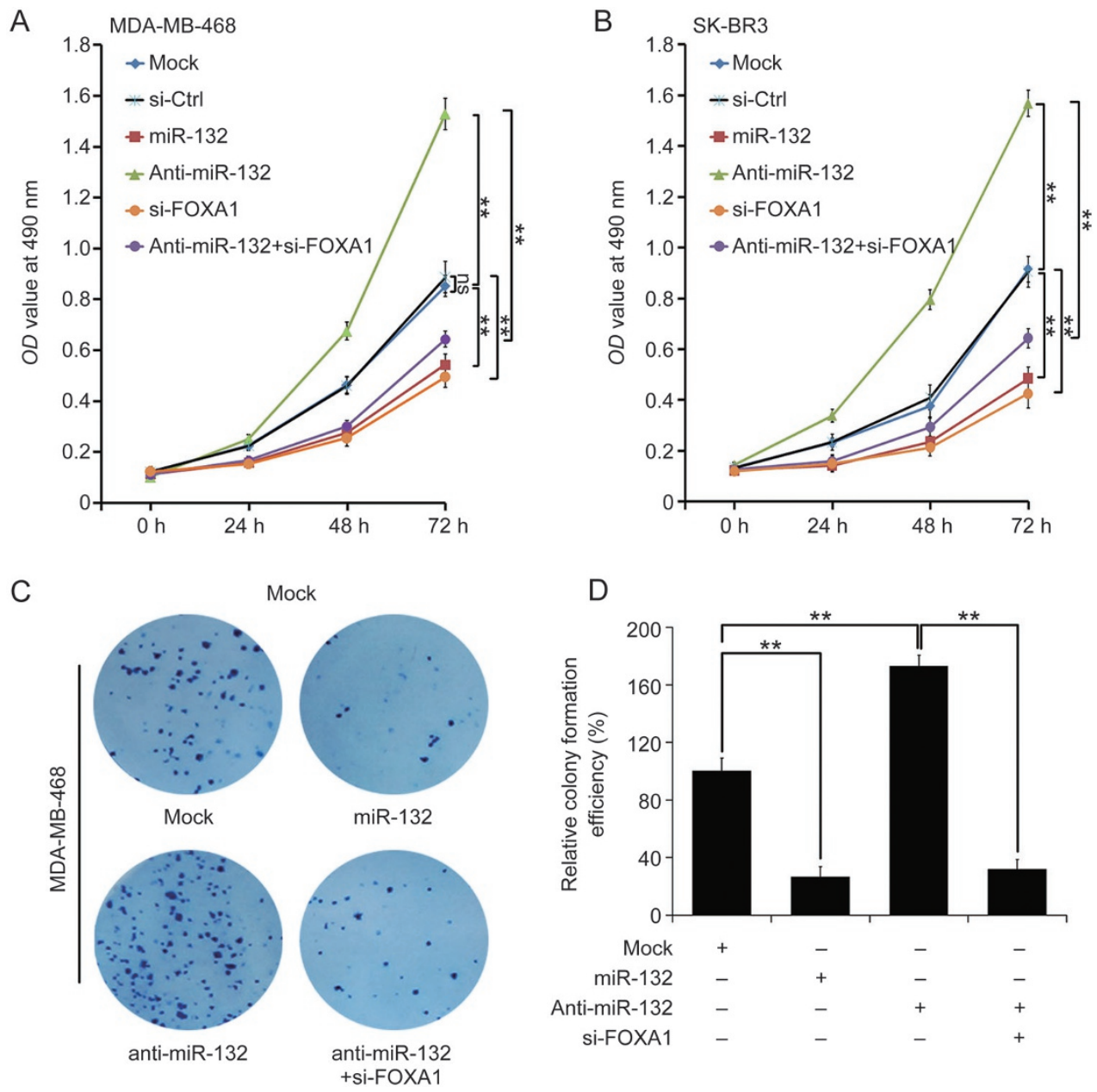

Figure 4. MiR-132 suppresses proliferation of breast cancer cells through FOXA1. (A and B) MTT assays demonstrated that miR-132 significantly inhibited the proliferation of MDA-MB-468 and SK-BR3, whereas anti-miR-132 significantly promoted the proliferation of MDA-MB-468 and SK-BR3. SiFOXA1 clearly decreased MDA-MB-468 and SK-BR3 cell proliferation. Furthermore, knockdown of FOXA1 by siRNA transfection reversed the effect of anti-miR-132-induced promotion of MDA-MB-468 and SK-BR3 proliferation. (C and D) Colony formation demonstrated that miR-132 significantly inhibited the colony formation efficiency of MDA-MB-468, whereas anti-miR-132 significantly promoted the colony formation efficiency of MDA-MB-468, an effect reversed by si-FOXA1. ${ }^{* *} P<0.01$.

cells and that the biological function of miR-132 in breast cancer is mediated by FOXA1, at least partially through inhibition FOXA1 expression.

\section{Discussion}

MicroRNAs have been implicated in cancer and play critical roles in cancer initiation and progression in cell proliferation, apoptosis, differentiation, invasion and metastasis ${ }^{[35-37]}$. Increasing evidence demonstrates that microRNAs are potential biomarkers or therapeutic targets in tumors. Therefore, the identification and complete characterization of cancerassociated miRNAs, including their direct targets, should greatly contribute to the ultimate goal of curing cancer in humans.

In this study, miR-132 was found to be negatively correlated with FOXA1 expression, which was significantly upregulated in breast cancer tissues compared with adjacent normal tissues. FOXA1 was predicted to be a direct target of miR-132, as validated by dual luciferase assays and Western blotting. Dual luciferase assays demonstrated that miR-132 significantly inhibited the luciferase activity of the wild-type of 3'UTR of FOXA1 rather than the mutant form. Western blotting demonstrated that ectopic miR-132 significantly inhibited the expression of FOXA1, whereas miR-132 knockdown significantly promoted FOXA1 expression in breast cancer cells. Furthermore, miR-132 played a critical role as a tumor suppressor in breast cancer. MiR-132 significantly inhibited cell proliferation and colony formation in breast cancer cells, whereas knockdown of miR-132 significantly promoted cell growth, which was attenuated by FOXA1 knockdown. Together, our results demonstrated that miR-132 suppresses the proliferation of breast cancer cells at least partially by directly targeting FOXA1.

MiR-132, which is part of the miR-212/132 cluster, plays an important role in inflammation, cell transformation, vascular smooth muscle and tumorigenesis ${ }^{[21-23,31]}$. Regarding cancer, 
downregulation of miR-132 and its functional role as a tumor suppressor has been reported in various cancer types, including breast cancer ${ }^{[21]}$, prostate cancer ${ }^{[26]}$, colorectal cancer ${ }^{[22]}$, osteosarcoma $^{[27]}$, non-small cell lung cancer ${ }^{[24,28]}$, and hepatocellular carcinoma ${ }^{[29-31]}$. However, miR-132 expression is upregulated in glioma and gastric cancer, and miR-132 might serve as an oncogene in these cancers ${ }^{[2,38]}$. These reports have demonstrated that dysregulation of miR-132 is cancer specific and suggest that the underlying mechanisms involved might be different. In our study, miR-132 was found to function as a tumor suppressor via downregulation of FOXA1.

FOXA1 is termed a "pioneer factor" because it binds condensed chromatin, thereby delineating genomic regions and allowing for subsequent binding of other transcription factors, such as the ER, progesterone receptor (PR) ${ }^{[39]}$ and androgen receptor $(\mathrm{AR})^{[40,41]}$. In hormone-related cancers, such as breast cancer and prostate cancer, chromatin opening induced by FOXA1 contributes to binding of the ER in breast cancer ${ }^{[42]}$ and the AR in prostate and breast cancers ${ }^{[9]}$. Together with ER, FOXA1 alters the expression patterns of gene transcription that induce luminal cell differentiation ${ }^{[43]}$ and repress the basal phenotype ${ }^{[6]}$. Thus, FOXA1 has a key role in demarcating the tissue-specific binding sites of these transcription factors ${ }^{[10]}$. However, a recent study demonstrated that FOXA1 is highly expressed in $\mathrm{ER}^{+}$breast cancer cells (MCF-7, T47D, BT474 and ZR75), ER ${ }^{-} / \mathrm{HER}^{+}{ }^{+}$SK-BR3 cells, and triple negative-like MDA-MB-468 cell lines. In all breast cancer subtypes, FOXA1 or FOXA2 control of the endothelial lipase LIPG is ubiquitous. In these subtypes, LIPG is highly expressed, and FOXA-mediated regulation of LIPG controls the uptake of extracellular lipids, thus supporting breast cancer growth ${ }^{[11]}$. Interestingly, the expression of LIPG, a downstream effector of FOXA1, was decreased by miR-132. Finally, we found that FOXA1 expression negatively correlated with miR-132 expression in breast cancer tissues and was inhibited by miR-132 in breast cancer cells. Thus, our findings provide important information and evidence to improve characterization of the miR-132/FOXA1 axis in breast cancer.

In summary, we demonstrated that miR-132 inhibits breast cancer cell proliferation at least partially via downregulation of FOXA1. Our findings suggest that dysregulation of miR132 might account for FOXA1 overexpression in breast cancer tissues, and modulation of miR-132 and FOXA1 may offer potential therapeutic targets in breast cancer.

\section{Author contribution}

Dan YU and Jin-ling FU conceived and designed the experiments. Dan WANG and Jin REN performed the experiments. Jin REN and Hui REN analyzed the data. Dan WANG and Jin-ling FU wrote the paper.

\section{References}

1 Jemal A, Bray F, Center MM, Ferlay J, Ward E, Forman D. Global cancer statistics. CA Cancer J Clin 2011; 61: 69-90.

2 Liu W, Schaffer L, Herrs N, Chollet C, Taylor S. Improved sleep after Qigong exercise in breast cancer survivors: A pilot study. Asia Pac J
Oncol Nurs 2015; 2: 232-9.

3 Kam J, Kam J, Mann GB, Phillips C, Wentworth JM, King J, et al. Solitary pituitary metastasis from HER2-positive breast cancer. Asia Pac J Clin Oncol 2017; 13: e181-e184. doi: 10.1111/ajco.12353.

4 Droog M, Nevedomskaya E, Kim Y, Severson T, Flach KD, Opdam M, et al. Comparative cistromics reveals genomic cross-talk between FOXA1 and ERalpha in tamoxifen-associated endometrial carcinomas. Cancer Res 2016; 76: 3773-84.

5 Hurtado A, Holmes KA, Ross-Innes CS, Schmidt D, Carroll JS. FOXA1 is a key determinant of estrogen receptor function and endocrine response. Nat Genet 2011; 43: 27-33.

6 Bernardo GM, Bebek G, Ginther CL, Sizemore ST, Lozada KL, Miedler JD, et al. FOXA1 represses the molecular phenotype of basal breast cancer cells. Oncogene 2013; 32: 554-63.

7 Mehta RJ, Jain RK, Leung S, Choo J, Nielsen T, Huntsman D, et al. FOXA1 is an independent prognostic marker for ER-positive breast cancer. Breast Cancer Res Treat 2012; 131: 881-90.

8 Nucera C, Eeckhoute J, Finn S, Carroll JS, Ligon AH, Priolo C, et al. FOXA1 is a potential oncogene in anaplastic thyroid carcinoma. Clin Cancer Res 2009; 15: 3680-9.

9 Yang YA, Yu J. Current perspectives on FOXA1 regulation of androgen receptor signaling and prostate cancer. Genes Dis 2015; 2: 144-51.

10 Fu X, Jeselsohn R, Pereira R, Hollingsworth EF, Creighton CJ, Li F, et al. FOXA1 overexpression mediates endocrine resistance by altering the ER transcriptome and IL-8 expression in ER-positive breast cancer. Proc Natl Acad Sci U S A 2016; 113: E6600-E09.

11 Slebe F, Rojo F, Vinaixa M, Garcia-Rocha M, Testoni G, Guiu M, et al. FoxA and LIPG endothelial lipase control the uptake of extracellular lipids for breast cancer growth. Nat Commun 2016; 7: 11199.

12 Cancer Genome Atlas N. Comprehensive molecular portraits of human breast tumours. Nature 2012; 490: 61-70.

13 Zhao J, Zhao Y, Wang Z, Xuan Y, Luo Y, Jiao W. Loss expression of micro ribonucleic acid (miRNA)-200c induces adverse post-surgical prognosis of advanced stage non-small cell lung carcinoma and its potential relationship with ETAR messenger RNA. Thorac Cancer 2015; 6: 421-6.

14 Mar-Aguilar F, Luna-Aguirre CM, Moreno-Rocha JC, Araiza-Chavez J, Trevino V, Rodriguez-Padilla C, et al. Differential expression of miR-21, miR-125b and miR-191 in breast cancer tissue. Asia Pac J Clin Oncol 2013; 9: 53-9.

15 Serpico D, Molino L, Di Cosimo S. microRNAs in breast cancer development and treatment. Cancer Treat Rev 2014; 40: 595-604.

16 Chen X, Lu P, Wang DD, Yang SJ, Wu Y, Shen HY, et al. The role of miRNAs in drug resistance and prognosis of breast cancer formalinfixed paraffin-embedded tissues. Gene 2016; 595: 221-6.

17 Zhang W, Xu J, Shi Y, Sun Q, Zhang Q, Guan X. The novel role of miRNAs for tamoxifen resistance in human breast cancer. Cell Mol Life Sci 2015; 72: 2575-84.

18 Venkatadri R, Muni T, lyer AK, Yakisich JS, Azad N. Role of apoptosisrelated miRNAs in resveratrol-induced breast cancer cell death. Cell Death Dis 2016; 7: e2104.

19 Yang $\mathrm{Y}$, Jiang G, Zhang P, Fan J. Programmed cell death and its role in inflammation. Mil Med Res 2015; 2: 12.

20 Wanet A, Tacheny A, Arnould T, Renard P. miR-212/132 expression and functions: within and beyond the neuronal compartment. Nucleic Acids Res 2012; 40: 4742-53.

21 Zhang ZG, Chen WX, Wu YH, Liang HF, Zhang BX. MiR-132 prohibits proliferation, invasion, migration, and metastasis in breast cancer by targeting HN1. Biochem Biophys Res Commun 2014; 454: 109-14.

22 Zheng YB, Luo HP, Shi Q, Hao ZN, Ding Y, Wang QS, et al. miR132 inhibits colorectal cancer invasion and metastasis via directly 
targeting ZEB2. World J Gastroenterol 2014; 20: 6515-22.

23 Li W, Zhang J, Chen T, Yin P, Yang J, Cao Y. miR-132 upregulation promotes gastric cancer cell growth through suppression of Fox01 translation. Tumour Biol 2015. [Epub ahead of print]. Doi: 10.1007/ s13277-015-3924-y.

24 Li Y, Zu L, Wang Y, Wang M, Chen P, Zhou Q. miR-132 inhibits lung cancer cell migration and invasion by targeting SoX4. J Thorac Dis 2015; 7: 1563-9.

25 Chen S, Wang Y, Ni C, Meng G, Sheng X. HLF/miR-132/TTK axis regulates cell proliferation, metastasis and radiosensitivity of glioma cells. Biomed Pharmacother 2016; 83: 898-904.

26 Qu W, Ding SM, Cao G, Wang SJ, Zheng XH, Li GH. miR-132 mediates a metabolic shift in prostate cancer cells by targeting Glut1. FEBS Open Biol 2016; 6: 735-41.

27 Wang J, Xu G, Shen F, Kang Y. miR-132 targeting cyclin E1 suppresses cell proliferation in osteosarcoma cells. Tumor Biol 2014; 35: 485965.

28 Zhang B, Lu L, Zhang X, Ye W, Wu J, Xi Q, et al. Hsa-miR-132 regulates apoptosis in non-small cell lung cancer independent of acetylcholinesterase. J Mol Neurosci 2014; 53: 335-44.

29 Lei CJ, Yao C, Li DK, Long ZX, Li Y, Tao D, et al. Effect of cotransfection of miR-520c-3p and miR-132 on proliferation and apoptosis of hepatocellular carcinoma Huh7. Asian Pac J Trop Med 2016; 9: 898-902.

30 Lei CJ, Li L, Gao X, Zhang J, Pan QY, Long HC, et al. Hsa-miR-132 inhibits proliferation of hepatic carcinoma cells by targeting YAP. Cell Biochem Funct 2015; 33: 326-33.

31 Wei X, Tan C, Tang C, Ren G, Xiang T, Qiu Z, et al. Epigenetic repression of miR-132 expression by the hepatitis $B$ virus $X$ protein in hepatitis B virus-related hepatocellular carcinoma. Cell Signal 2013; 25: 1037-43.

32 Zhang S, Hao J, Xie F, Hu X, Liu C, Tong J, et al. Downregulation of miR-132 by promoter methylation contributes to pancreatic cancer development. Carcinogenesis 2011; 32: 1183-9.

33 Formosa A, Lena AM, Markert EK, Cortelli S, Miano R, Mauriello A, et al. DNA methylation silences miR-132 in prostate cancer. Oncogene 2013; 32: 127-34.

34 Damavandi Z, Torkashvand S, Vasei M, Soltani BM, Tavallaei M, Mowla SJ. Aberrant expression of breast development-related microRNAs, miR-22, miR-132, and miR-212, in breast tumor tissues. J Breast Cancer 2016; 19: 148-55.

35 Takashima Y, Terada M, Udono M, Miura S, Yamamoto J, Suzuki A. Suppression of lethal- $7 \mathrm{~b}$ and miR-125a/b maturation by Lin28b enables maintenance of stem cell properties in hepatoblasts. Hepatology 2016; 64: 245-60.

36 Powers JT, Tsanov KM, Pearson DS, Roels F, Spina CS, Ebright R, et al. Multiple mechanisms disrupt the let-7 microRNA family in neuroblastoma. Nature 2016; 535: 246-51.

37 Lv P, Zhang P, Li X, Chen Y. Micro ribonucleic acid (RNA)-101 inhibits cell proliferation and invasion of lung cancer by regulating cyclooxygenase-2. Thorac Cancer 2015; 6: 778-84.

38 Liu Q, Liao F, Wu H, Cai T, Yang L, Wang ZF, et al. Upregulation of miR132 expression in glioma and its clinical significance. Tumor Biol 2014; 35: 12299-304.

39 Clarke CL, Graham JD. Non-overlapping progesterone receptor cistromes contribute to cell-specific transcriptional outcomes. PLoS One 2012; 7: e35859.

40 Jozwik KM, Carroll JS. Pioneer factors in hormone-dependent cancers. Nat Rev Cancer 2012; 12: 381-5.

41 Jozwik KM, Chernukhin I, Serandour AA, Nagarajan S, Carroll JS. FOXA1 directs H3K4 monomethylation at enhancers via recruitment of the methyltransferase MLL3. Cell Rep 2016; 17: 2715-23.

42 Carroll JS, Liu XS, Brodsky AS, Li W, Meyer CA, Szary AJ, et al. Chromosome-wide mapping of estrogen receptor binding reveals longrange regulation requiring the forkhead protein FoxA1. Cell 2005; 122: 33-43.

43 Ademuyiwa FO, Thorat MA, Jain RK, Nakshatri H, Badve S. Expression of Forkhead-box protein A1, a marker of luminal A type breast cancer, parallels low oncotype DX 21-gene recurrence scores. Mod Pathol 2010; 23: 270-5. 